\title{
TENDENCIAS ESTACIONALES DE LA PRECIPITACIÓN EN LA CUENCA DEL EBRO 1951-2000*
}

\author{
De Luis, M. (1), Longares, L.A. (1), Stepanek, P. (2) \\ y González-Hidalgo, J.C. (1) \\ (1) Departamento de Geografía, Universidad de Zaragoza, 50009 Zaragoza, España \\ (2) Czech Hydrometeorological Institute, Brno, Czech Republic \\ e-mail: mailto: mdla@unizar.es
}

\begin{abstract}
Resumen: Hemos analizado las tendencias de la precipitación anual y estacional de la cuenca del Ebro durante la segunda mitad del siglo XX (1951-2000) con todos los observatorios procedentes de la base de datos de precipitaciones mensuales de la vertiente mediterránea española $\left(\right.$ MOPREDA $\left._{\mathrm{MES}}\right)$. El total de series analizadas es de 424, lo que supone una densidad promedio de 1 observatorio cada $200 \mathrm{~km}^{2}$, incluyendo información hasta $1250 \mathrm{~m}$ de altitud. Los resultados permiten sugerir que existe un ligero y generalizado descenso de los totales anuales de precipitación que afecta a 3/4 del total de observatorios. Estacionalmente el verano es la estación que muestra un descenso significativo más generalizado ( $44 \%$ de observatorios). En invierno, primavera y otoño el descenso afecta a $1 / 3$ del total. Sin embargo en el espacio el comportamiento de cada estación difiere. Durante el invierno el descenso es generalizado (aunque no significativo), en primavera y verano se detectan ligeros incrementos de precipitación en la cabecera de la cuenca, mientras en la margen izquierda se localiza un área donde predominan las pérdidas con carácter significativo. El otoño permite establecer una clara distinción entre la margen izquierda, con tendencia positiva, y la margen derecha, con tendencia negativa.
\end{abstract}

Palabras clave: Precipitación, tendencia, análisis estacional, cuenca del Ebro, España.

Abstract: We have analyzed seasonal and annual precipitation trend in the Ebro catchment (NE Spain) during the second half of XXth (1951 2000). Data analyzed come from monthly precipitation data base of Mediterranean Spain (MOPRE$\mathrm{DA}_{\mathrm{MES}}$ ). The total amount of rainfall series is 424 and the mean value of spatial density is 1 observatory per $200 \mathrm{~km}^{2}$; data base include information in altitude until $1250 \mathrm{~m}$ osI. The results allow us to suggest that in the Ebro catchment

* Recibido: 25-10-07. Aceptado: 14-12-07. 
during the second half of the XXth century annual precipitation has slight decreased in 3/4 of total stations. At seasonal scale the most generalized decreasing of precipitation is found during summer ( $44 \%$ of total stations). Significant decreasing affects $1 / 3$ of total stations in winter, spring and fall. Spatial differences can be described. In winter precipitation shows a generalized negative trend but mainly not significant. During spring and summer we have detected slight increment in the upper catchment area, while left margin of the Ebro catchment has been under negative and significant trend. A clear difference exits from $\mathrm{N}$ to $\mathrm{S}$ during autumn, with positive and negative trend respectively.

Keyword: Precipitation. Trend. Seasonal analysis. Annual analysis. Ebro catchment. Spain.

\section{Introducción}

El reciente informe del IPCC 2007 (en adelante AR4) ha confirmado un incremento de las precipitaciones durante el periodo 1900-2005 sobre las tierras emergidas al norte del paralelo $30^{\circ}$ (coherentemente con el calentamiento detectado), su descenso desde 1970 en torno a los trópicos, y el aumento de las áreas afectadas por la sequía en la zona tropical y subtropical desde dicha fecha (Trenberth et al., 2007). De modo más concreto la precipitación en la franja subtropical del hemisferio norte $\left(20^{\circ}-40^{\circ} \mathrm{N}\right)$ no ha mostrado una señal clara de tendencias a lo largo del siglo XX, y se ha caracterizado por una fuerte variabilidad entre décadas (New et al., 2001). Esta zona latitudinal comprende la cuenca mediterránea, área de transición climática en la que se ha sugerido que se sentirán con mayor rapidez los efectos del cambio global (Lavorel et al., 1996; Esteban Parra et al., 1998). Los modelos climáticos predicen para finales del siglo XXI en el entorno del Mediterráneo un descenso de los totales anuales y un aumento de la variabilidad de las precipitaciones (Christensen et al., 2007, p. 874877). Sin embargo, estos resultados presentan una gran incertidumbre por la gran variabilidad que caracteriza las precipitaciones del área mediterránea (Lionello et al., 2006). En este contexto se entiende la recomendación del AR4 de realizar estudios subregionales sobre la variabilidad de las precipitaciones. Para ello se requieren bases de datos con la mayor densidad espacial posible de observaciones (Lana y Burgueño, 2000; Huntington, 2006; Trenberth et al., 2007), no siembre disponibles.

Los estudios sobre precipitaciones en Península Ibérica son numerosísimos aunque De Castro et al. (2006) han indicado que carecemos aún de un análisis global pormenorizado por la ausencia de información detallada. Tradicionalmente la Península se divide en tres dominios pluviales: el norte húmedo, el centro-sur y la fachada mediterránea, pero no se debe olvidar que esta división esconde numerosos matices y variaciones espaciales que sólo se pueden descubrir mediante el análisis 
subregional, para lo que es necesario contar con una elevada densidad de observaciones. La latitud, el relieve y la peculiar posición geográfica de la Península Ibérica, entre dos masas de agua contrastadas, han dificultado los análisis espaciales de detalle (Lana y Burgueño, 2000; Sumner et al., 2001; Goodess y Jones, 2002; Martin Vide, 2004).

En este trabajo analizamos las tendencias de la precipitación anual y estacional de un sector de la fachada mediterránea española, la cuenca del Ebro, durante la segunda mitad del siglo XX (1951 2000), El estudio emplea series de precipitación mensual de

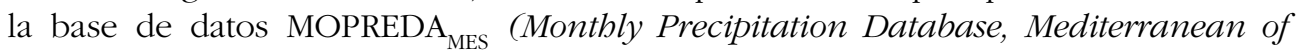
Spain). La citada base de datos ofrece en el momento actual la mayor densidad de observatorios durante la segunda mitad del siglo XX no sólo de todo el entorno mediterráneo y de España, sino evidentemente de la cuenca analizada.

\section{2. Área de estudio y métodos}

\section{1. Área de estudio}

La cuenca del Ebro se localiza en el cuadrante nororiental de la Península Ibérica, donde se dispone con grosera forma de triángulo isósceles en sentido NW-SE (Figura 1). Su extensión es de $86.000 \mathrm{~km}^{2}$, aproximadamente, quedando delimitada por la divisoria de aguas de los Pirineos al norte, las cordilleras Ibéricas por el Oeste y la cordillera costero catalana al este, abriéndose al Mediterráneo por el estrecho corredor del Bajo Ebro. Incluye territorios de las Comunidades Autónomas del País Vasco, Cantabria, Castilla y León, la Rioja, Navarra, Aragón, Cataluña, Castilla la Mancha y Comunidad Valenciana, así como El Principado de Andorra y un pequeño territorio francés, si bien Aragón engloba prácticamente el 50 \% del espacio.

Por la posición latitudinal, por su disposición NW-SE, y por su localización interior en la Península Ibérica, este espacio podría calificarse como "la transición de la transición", pues desde su nacimiento en Fontibre, en la falda sur de los montes Cantábrico, hasta su desembocadura en Tortosa, casi mil kilómetros hacia el E-SE, la variación climática es notable e incluye condiciones cuasi oceánicas al NW, la alta montaña pirenaica, o los semidesiertos de Bardenas y bajo Aragón.

\subsection{Base de datos de precipitaciones mensuales de la cuenca del Ebro}

Los datos empleados en este estudio son las series mensuales de precipitación procedentes de la base de datos de la vertiente mediterránea española, MOPREDA ${ }_{\mathrm{MES}}$ 


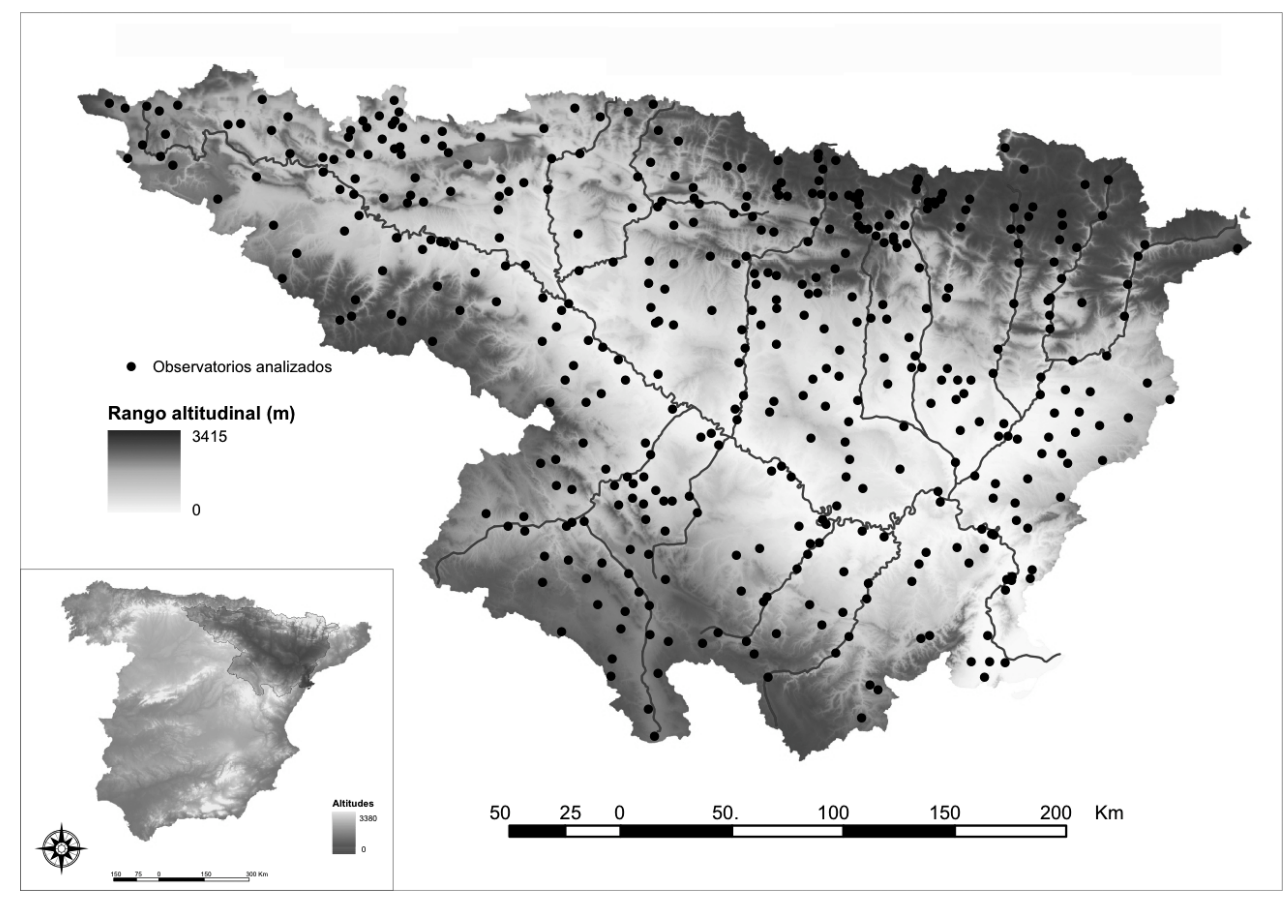

Figura 1. Localización de la cuenca del Ebro y observatorios empleados procedentes de la base de datos MOPREDA MES $_{\text {. }}$

(González Hidalgo et al., 2006). Esta base de datos se ha elaborado analizando el total de registros almacenados en los archivos del INM. En el caso particular de la cuenca del Ebro se conservan al menos datos de precipitación mensual de 1598 observatorios, de los que 1130 proceden de series con más de 10 años de registros.

El proceso de control de calidad ante la ausencia de metadatos disponibles, se realizó en primer lugar tratando de conseguir un conjunto de series de referencia de vecinos de la máxima calidad; seguidamente se identificaron y eliminaron todos los datos considerados anómalos, y finalmente se contrastó la homogeneidad de cada serie. Por ultimo con las series libres de datos anómalos y de inhomogeneidades, se efectuó un proceso de reconstrucción, empleando en este trabajo las series del periodo 1951 2000. El control de calidad y la reconstrucción se realizaron por medio de un proceso iterativo de confección de sucesivas series de referencia que se describe en detalle a continuación. 


\subsubsection{Las series de referencia}

Existe un acuerdo generalizado que asume que el éxito de cualquier control de calidad de datos climáticos, así como cualquier reconstrucción climática a partir de series fragmentadas, depende en gran medida de la calidad de las series de referencia que se empleen, por lo que estas son un punto central en todo estudio de datos climáticos (Begert et al., 2005). Existe también un acuerdo general sobre cómo seleccionar los observatorios vecinos para construir una serie de referencia de cualquier observatorio (elevada correlación y cercanía), pero sin embargo hay diferentes puntos de vista sobre cómo calcular dichas series (véase p.e. Michell y Jones, 2005; Auer et al., 2005; Brunetti et al., 2006). En principio los observatorios vecinos deben estar correlacionados positivamente con el observatorio candidato, superando un umbral definido entre $r>0.7$ y r $>0.8$ (Vincent y Gullet, 1999; Auer et al., 2005), que en ocasiones se ha acortado ( $r>0.4$ ) (Mitchell y Jones, 2005), lo que no debe olvidarse en áreas de clima mediterráneo donde la correlación entre vecinos puede ser muy baja especialmente en verano (Rodríguez et al., 1999). Por otro lado para evitar los efectos de los datos extremos (Lanzante, 1996; González Rouco et al., 2001) dicha correlación se ha sugerido que debería calcularse a partir de las series de diferencias o ratios (Peterson et al., 1998a), o tras una transformación logarítmica (Rhoades y Salinger, 1993). Por último no hay acuerdo sobre el número de vecinos que deben considerarse (Peterson y Easterling, 1994; Keiser y Griffiths, 1997; Rhoades y Salinger, 1993).

El segundo criterio, la distancia, es lógico si tenemos en cuenta que una serie de referencia debe mostrar el comportamiento general del área en que se localiza el observatorio estudiado. Obviamente los observatorios vecinos deberían estar cerca del observatorio analizado, sin obstáculos intermedios como montañas y barreras que modifiquen su comportamiento (Vincent y Gullet, 1999). Brunetti et al. (2006) en Italia, y Auer et al. (2005) en los Alpes han sugerido que dicha distancia no debería superar los $100 \mathrm{~km}$.

El paso final de una serie de referencia es la elección del algoritmo de cálculo. Por lo general una serie de referencia se calcula mediante una media ponderada entre vecinos, utilizando como factor de ponderación el coeficiente de correlación o la distancia (ejemplos diferentes se muestran en Alexandersson, 1986 y 2001; Peterson y Easterling, 1994; Peterson et al., 1998 a y b).

En la base de datos MOPREDA $_{\mathrm{MES}}$ hemos adoptado el siguiente procedimiento general para seleccionar los vecinos de las series de referencia. En primer lugar, sobre una matriz de correlaciones mensuales de todos los observatorios se descartaron las parejas de correlaciones (esto es, vecinos) con solapamiento inferior a 10 años, así como todos los casos en los que al menos un mes presentase valores negativos, o, 
siendo todos los meses positivamente correlacionados, el promedio de los doce meses fuese inferior a 0,50. A continuación, a partir de la matriz de correlaciones depurada se calcularon las series de referencia a $25 \mathrm{~km}, 50 \mathrm{~km}$ y $75 \mathrm{~km}$ (procedimiento descrito más adelante). A $<25 \mathrm{~km}$ en muchos casos la serie de referencia resultó incompleta por la ausencia de vecinos durante determinados años, mientras que las series de referencia a $75 \mathrm{~km}$ de distancia se correlacionaron en menor medida que a $50 \mathrm{~km}$, por lo cual la distancia final seleccionada fue de $50 \mathrm{~km}$. En resumen, las series de referencia que hemos empleado se calcularon a partir de todos los vecinos correlacionados positivamente durante todos los meses, cuyo promedio de correlaciones mensuales fuera $r>0.50$, tuviesen registros solapados al menos 10 años con el observatorio candidato, y no distantes más de $50 \mathrm{~km}$.

Con este procedimiento se pudo aprovechar una gran cantidad de información contenida en los archivos del INM, de hecho toda la referente a series con más de 10 años de registros, y nos permitió producir series de referencia para todos los observatorios analizados. Sin embargo el proceso tiene un inconveniente pues las series de referencia a lo largo de los años pueden tener diferente número de vecinos (cosa que ocurre con mucha frecuencia). Para evitar este hecho los datos de cada observatorio vecino (recordemos solapados 10 años al menos con el observatorio candidato) se transformaron aplicando como factor de corrección a cada serie vecina el valor de la ratio de los promedios del periodo común con el observatorio candidato. El cálculo final de las series de referencia se realizó mediante una media ponderada con coeficiente de ponderación $\left(1 / \mathrm{d}^{2}\right)$ siendo "d" la distancia de cada vecino.

\subsubsection{Detección de datos sospechosos}

La detección de datos anómalos ha sido recientemente señalada como un aspecto clave de la construcción de bases de datos climáticas (Auer et al., 2005; González Rouco et al., 2001), capaz de poder afectar a la identificación de inhomogeneidades

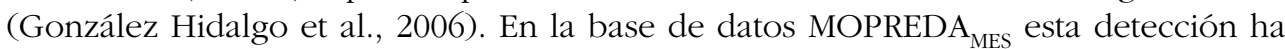
tenido dos objetivos: en primer lugar asegurar la calidad de la serie de referencia y en segundo lugar depurar los datos originales.

Las precipitaciones mensuales en la Península Ibérica pueden alcanzar cantidades francamente extraordinaria y presentar una gran variabilidad espacial. Las dos consideraciones nos han sugerido adoptar los procedimientos comunes de detección de datos anómalos a nuestra realidad combinando dos criterios: las ratios y la distancia intercuartílica. En el primer caso una ratio nos informa de la diferencia entre la serie candidata y su referencia en un dato relativo a un año y mes. En el segundo caso, el análisis intercuartílico aplicado a las series de ratios nos indica la relación del valor de cada ratio en el contexto temporal de una serie candidata y su referencia, es decir si la diferencia en un mes y año es persistente o no. El exceso sobre el rango inter- 
cuartílico (IQRE) de cada ratio se calcula según (q75+((q75 q25)*3)), con q75 el cuartil 3, q25 el cuartil 1, y 3 un coeficiente (González Rouco et al., 2001). Finalmente, debido a que las series de precipitación se encuentran acotadas por su base (mínimo por defecto es 0), ambos procedimientos de detección se aplicaron sobre las series de ratios directas (Candidata/Referencia) e inversas (Referencia/Candidata). La identificación de un valor como sospechoso combinó diferentes umbrales de ratios y coeficientes de exceso intercuartílicos. Mayores especificaciones, detalles y ejemplos se encuentran en González Hidalgo et al. (2006).

Los datos considerados anómalos fueron eliminados de la base de datos y procedimos a recalcular una nueva serie de referencia. El proceso se iteró hasta que ningún dato fue calificado de sospechoso tras 10 iteraciones. Llegados a este punto, las series de referencia se consideraron provisionalmente como las de mayor calidad disponible, siendo contrastadas de nuevo con los datos originales sin depurar para efectuar la detección definitiva de datos anómalos. El total de datos finalmente descartados supuso menos del $1 \%$ del total.

El proceso es conservador, y sin duda alguna somos conscientes de que diversos datos anómalos se han conservado, aunque opinamos que al menos los datos descartados deben ser eliminados del análisis.

\subsubsection{Análisis de Homogeneidad}

El segundo paso del control de calidad consistió en la verificación de la homogeneidad de las series depuradas de datos anómalos. A partir de las series depuradas de datos anómalos calculamos una nueva tanda de series de referencia y aplicamos el Standard Normal Homogeneity Test (SNHT) (Alexandersson, 1986). Esta prueba estadística asume que la precipitación en el observatorio candidato mantiene una proporcionalidad con la de la serie de referencia. Alexandersson (1986), Keiser y Griffiths (1997), y González Rouco et al. (2001) ofrecen explicaciones de la misma, y en González Hidalgo et al. (2002) se muestra un ejemplo pautado del cálculo. Brevemente, el SNHT parte de calcular la serie de ratios, q(i), según (1):

$$
\mathrm{q}(\mathrm{i}),=\mathrm{C}(\mathrm{i}) / \mathrm{R}(\mathrm{i}),(1 \leq \mathrm{i} \leq \mathrm{n})
$$

con C(i) el observatorio candidato y R(i) su referencia. A continuación la serie de ratios se estandariza según (2):

$$
z(i)=[q(i)-\bar{q}] / s,(1 \leq i \leq n)
$$


siendo $\bar{q}$ el promedio de q(i), o serie de ratios, y "s" su desviación. De este modo dos series de promedios $\bar{z}_{1}$ y $\bar{z}_{2}$ se pueden calcular, antes y después de cada uno de los valores de $z$. Finalmente las dos series se combinan en el estadístico $T_{(v)}(3)$ :

$$
T_{(v)}=\left[v \bar{z}_{1}^{2}+(\bar{n}-v) z_{1}^{2}\right],(1 \leq i \leq n)
$$

donde nuevamente " $\mathrm{v}$ " varía y "n" es el número de valores de la serie de ratios. El estadístico máximo $\mathrm{T}_{(\mathrm{v})}$ indica la máxima inhomogeneidad que existe en la serie respecto a sus valores críticos, recientemente tabulados por Khaliq y Ouarda (2007).

La prueba detecta inhomogeneidades estadísticas, por lo que para aceptar y corregir una serie empleamos ventanas móviles estudiando la acumulación de detecciones antes de proceder a corrección alguna. Una vez una inhomogeneidad se identifico como cierta, los datos anteriores al año de detección se corrigieron multiplicando por el factor $\left(q_{d} / q_{b}\right)$ siendo $q_{a}$ y $q_{b}$ los promedios de q(i) después (a) y antes (b) de la inhomogeneiedad. La prueba SNHT se aplicó en dos tandas sucesivas.

La reconstrucción de las series no completas durante 1951-2000 se efectuó con un nuevo conjunto de series de referencia calculadas sobre los datos ya homogeneizados. En este caso el cálculo empleó vecinos a menos de $10 \mathrm{~km}$, y para rellenar las lagunas resultantes utilizamos una segunda batería de referencias calculadas con vecinos a $25 \mathrm{~km}$ de distancia.

La selección final de observatorios reconstruidos para proceder a su análisis tuvo en cuenta el porcentaje de datos originales en el periodo 1951-2000, el porcentaje de datos reconstruidos a $10 \mathrm{~km}$, y la proporción de rellenados a $25 \mathrm{~km}$. Mas detalles se encuentran en González Hidalgo et al. (2006 a). En todo el proceso se empleó el software específicamente desarrollado para análisis climático Andim y ProclimDB (Stepanek 2006, a y b). En La Figura 1 se muestra la distribución espacial de los observatorios reconstruidos durante el periodo 1951-2000.

\subsection{Análisis de datos}

En cada observatorio se calcularon los promedios anuales y estacionales. Los meses incluidos en cada estación climática han seguido la convención climatológica adoptada por numerosos autores (Rodríguez Puebla et al., 1998; Romero et al., 1998), en la que invierno comprende (D-E-F), primavera (M-Ab-My) y sucesivamente el verano (J-JI-Ag) y otoño (S-O-N). Por la misma razón los valores anuales siempre incluyen el mes de diciembre del año previo. 
Para estimar la tendencia de las series de precipitaciones anuales y estacionales aplicamos la prueba de rangos de Spearman (Yue et al. 2002), según (4):

$$
\mathrm{r}_{\mathrm{s}}=\frac{1-\left[6 \sum\left(y_{i}-i\right)^{2}\right]}{n\left(n^{2}-1\right)}
$$

con "n" el número de datos de la serie (años) e "i" el orden del elemento de la serie original (el rango). La prueba se aplicó tras suavizar los datos originales con un filtro de paso bajo, lag 9. La probabilidad de trabajo empleada fue de $p<0.05$, que es el umbral seleccionado para asignar la posterior significación de las tendencias en el texto. La intensidad de los cambios observados de los valores de tendencia se analizó mediante regresiones lineales, tomando el valor del coeficiente b (pendiente de la recta) como indicador de la magnitud.

La distribución espacial de los resultados se muestra en una colección cartográfica donde se interpola el valor de la magnitud del cambio (coeficiente del modelo paramétrico), y se superpone el signo de tendencia resultante de la prueba de Spearman. La interpolación se realizó empleando $\left(1 / \mathrm{d}^{2}\right)$, con d la distancia al vecino más próximo.

\section{Resultados}

\subsection{Aspectos generales de la base de datos de precipitaciones mensuales MOPREDA $_{\text {MES EBRO }}$}

La historia de la información pluviométrica de la cuenca del Ebro se sintetiza en la Figura 2 donde mostramos la evolución anual del total de observatorios y la distancia promedio entre los mismos cada año. El desarrollo de la red de observaciones durante los primeros veinte años del siglo pasado, los efectos de la guerra, la rápida recuperación durante la postguerra y el declive de observaciones durante los últimos 25 años son hitos que quedan perfectamente señalados en la figura. Del mismo modo se observa que los registros comenzaron a mediados del siglo XIX, aunque solamente 5 observatorios registraban de manera más o menos continua datos de precipitación hasta bien entrada la primera década del siglo XX. Destaca poderosamente también que la distancia promedio entre observatorios (una medida de la densidad) se lograse de manera muy rápida, e incluso el impacto de la guerra, pese al cese de muchos observatorios, no afectase demasiado a dicho parámetro que prácticamente se mantuvo sin variación y rápidamente se recuperó. Sin duda alguna este hecho prueba la 
lógica de la planificación con que se desarrolló la toma de datos por el servicio meteorológico de aquellos momentos.

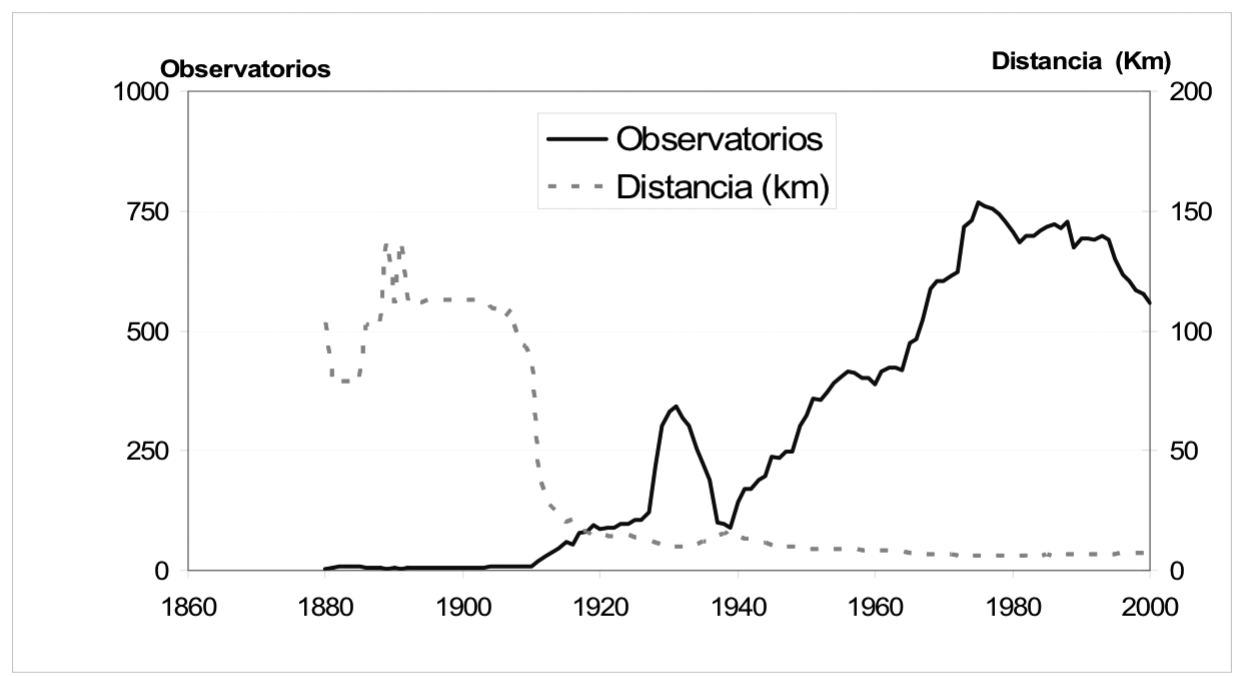

Figura 2. Evolución anual del número de observatorios con registros y promedio de distancia mínima (km).

En su conjunto MOPREDA ${ }_{M E S E B R O}$ proporciona una información de gran valor por la densidad espacial lograda en la cuenca del Ebro. Se han logrado reconstruir 71 series mensuales de precipitación completas durante el periodo 1911 2000; el total de series durante el periodo 1931-2000 es de 172, y durante la segunda mitad del siglo XX (1951-2000) las series resultaron ser 424. Este monto de series proporciona una densidad global en torno a 1 observatorio cada $200 \mathrm{~km}^{2}$ (véase Figura 1) y constituye el total de observatorios analizados en el presente trabajo.

La distribución de observatorios en el territorio de la cuenca por intervalo de altitudes se muestra en la Tabla 1. Como se puede apreciar la información es muy densa hasta alturas en torno a $1250 \mathrm{~m}$, lo cual supone aportar una información prácticamente ausente hasta el día de hoy. Incluimos el porcentaje de territorio de la cuenca en cada intervalo para poder ponderar la densidad espacial lograda.

\subsection{Distribución anual y estacional de las precipitaciones}

El reparto de las precipitaciones en la cuenca del Ebro es bien conocido, y los promedios de precipitación anuales y estacionales obtenidos con la base de datos 
Tabla 1. Distribución altitudinal de los observatorios de la base de datos MOPREDA MES EBRO, $_{\text {, }}$ periodo 1951-2000, porcentaje por intervalos de altitud, y porcentaje de territorio de la cuenca del Ebro.

\begin{tabular}{lccccccc}
\hline Altitudes & $<250$ & $250-500$ & $500-750$ & $750-1000$ & $1000-1250$ & $1250-1500$ & $>1500$ \\
Observatorios & 41 & 138 & 110 & 77 & 46 & 7 & 5 \\
Observatorios (\%) & 9.7 & 32.5 & 25.9 & 18.2 & 10.8 & 1.7 & 1.2 \\
Territorio (\%) & 8.3 & 25.7 & 21.4 & 19.0 & 13.6 & 5.3 & 6.7 \\
\hline
\end{tabular}

MOPREDA $_{M E S E B R O}$ muestran bien el conocido reparto de precipitaciones en el NE peninsular: máximos pirenaicos anuales $(>1000 \mathrm{~mm}$ ) y estacionales $(>300 \mathrm{~mm})$, que acaso se prolongan ocasionalmente en el extremo NW de la cabecera de la cuenca por las sierras Ibéricas del norte de Burgos y la Rioja salvo en el verano (nunca más al sur del Moncayo), y por el bajo Ebro en otoño, nunca más al oeste de las Garrigas. Los mínimos se corresponden con el sector central de la cuenca, a lo largo del eje del río y particularmente localizados en el entorno de la Bardena Navarra y el Bajo Aragón, donde las cantidades anuales oscilan entre 200 y $300 \mathrm{~mm}$ de promedio (Figura 3 y 4 ).

Esta distribución sugiere un cambio general de las condiciones productoras de precipitación a lo largo de las estaciones, predominando los flujos del N-NE durante el invierno y la primavera, que se combinan con los de procedencia E en el otoño, configurándose la cuenca como el lugar donde confluyen los efectos de diferentes modos de baja variabilidad (González Hidalgo et al., 2007; de Luis et al., 2007).

En conjunto los promedios tanto estacionales como anuales muestran una clara asimetría entre las márgenes montañosas izquierda (Pirineos) y derecha (Sistema Ibérico central y ramales meridionales), con valores que casi doblan los primeros a los segundos (Figura 4).

El régimen de precipitaciones predominante en la divisoria montañosa del norte es el invernal, el de primavera predomina a lo largo de la Ibérica, mientras en grandes extensiones del sector central de la cuenca hasta prácticamente la ciudad de Logroño la estación con mayores aportes es sorprendentemente el otoño, pese a que el mes más lluvioso es mayo. Este hecho pone de relevancia la necesidad de combinar los análisis estacionales y mensuales para alcanzar un conocimiento realista de la estructura temporal de las precipitaciones en la cuenca, y de este modo poder aplicar las conclusiones en el ámbito de la gestión de los recursos hídricos. 


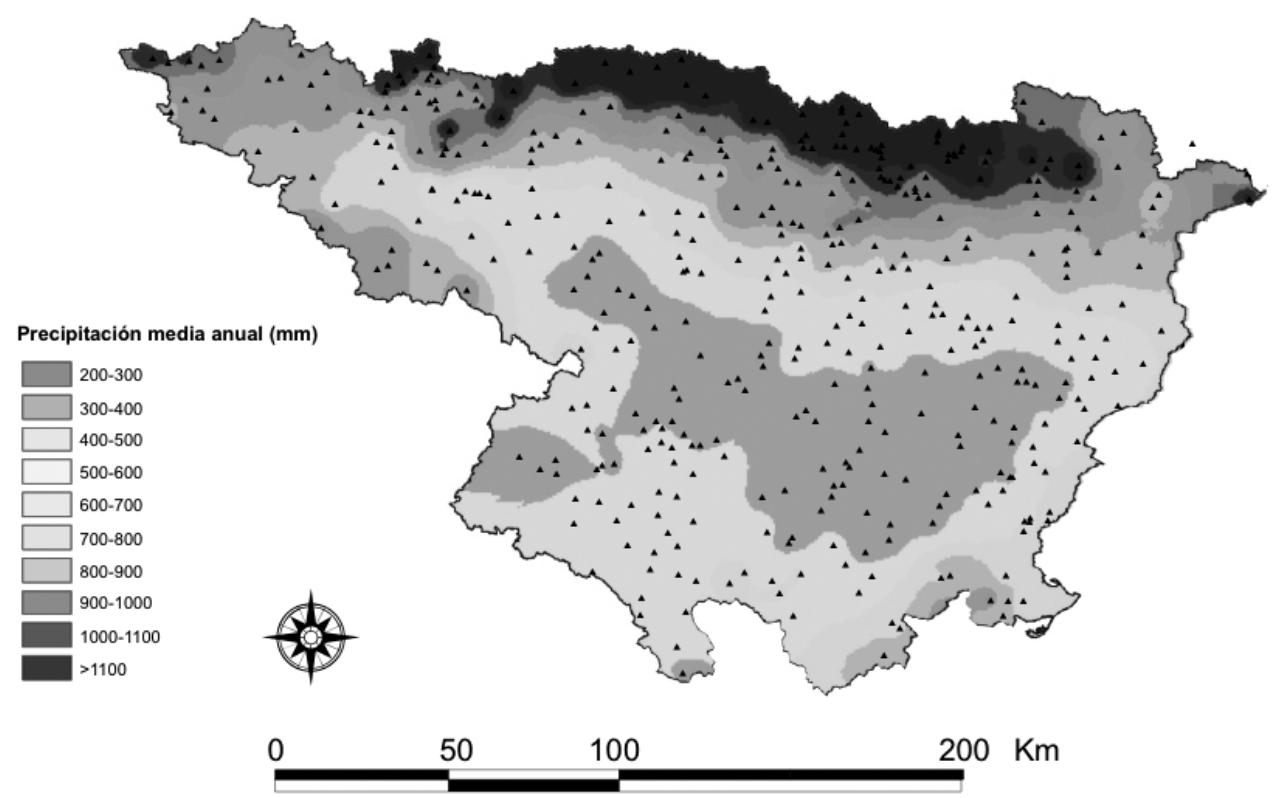

Figura 3. Promedio de precipitación anual $(\mathrm{mm})$ en la cuenca del Ebro.

\subsection{Tendencias estacionales y anuales}

Los resultados generales del análisis de tendencias se muestran en la Tabla 2, en la que aparece el total de observatorios por signo de tendencia (y su proporción) y el número de observatorios con tendencia significativa (y su proporción). En general predominan las tendencias de signo negativo en el total anual (93\% de observatorios), con un $63.9 \%$ de observatorios con tendencia significativa (Tabla 2). Estacionalmente también predominan las tendencias negativas. El invierno destaca por el alto porcentaje de observatorios afectados (90\%). Verano y otoño le siguen a cierta distancia (75\% de observatorios afectados) y la estación con menor número de observatorios con tendencia negativa es la primavera, en la que solamente se detecta un $64 \%$ de observatorios con tendencias negativas, mientras el $35 \%$ de observatorios presentan signo positivo.

Al considerar solamente las tendencias significativas el verano es la estación donde se observa un mayor número de observatorios con signo negativo (44\%), y algo menor es la proporción de observatorios afectados en invierno, primavera y otoño. 


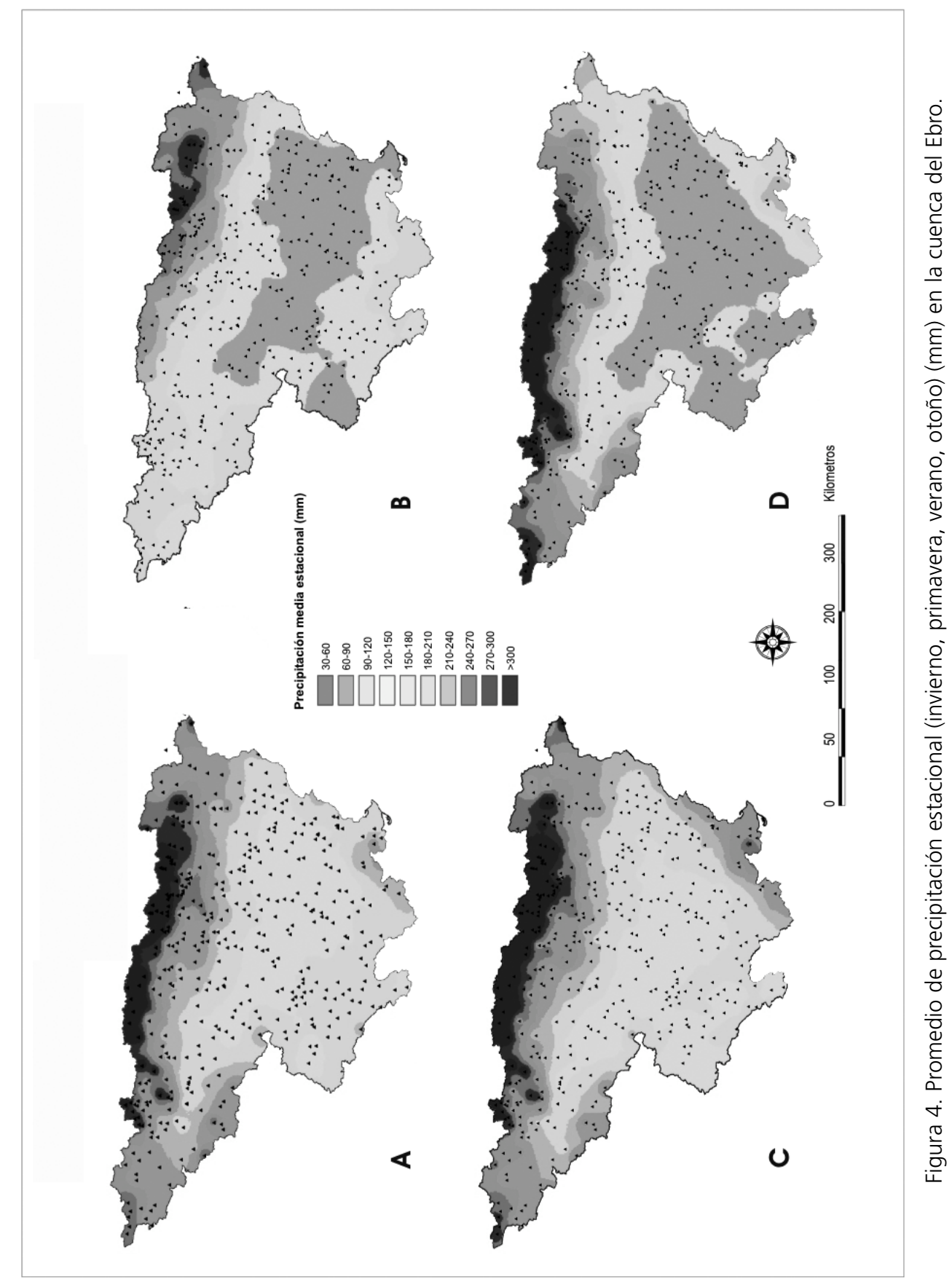


Tabla 2. Número de observatorios total según signo de tendencia, y observatorios con tendencias significativas $(p<0.05)$. Prueba de Spearman.

\begin{tabular}{|c|c|c|c|c|c|c|}
\hline & & Invierno & Primavera & Verano & Otoño & Año \\
\hline \multirow{4}{*}{ 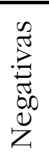 } & Total & 382 & 275 & 319 & 320 & 396 \\
\hline & $(\%)$ & 90.1 & 64.9 & 75.2 & 75.5 & 93.4 \\
\hline & Significativas & 140 & 135 & 188 & 136 & 271 \\
\hline & $(\%)$ & 33.0 & 31.8 & 44.3 & 32.1 & 63.9 \\
\hline \multirow{4}{*}{ : } & Total & 42 & 149 & 105 & 104 & 28 \\
\hline & $(\%)$ & 9.9 & 35.1 & 24.8 & 24.5 & 6.6 \\
\hline & Significativas & 2 & 27 & 36 & 7 & 7 \\
\hline & $(\%)$ & 0.5 & 6.4 & 8.5 & 1.7 & 1.7 \\
\hline
\end{tabular}

Destacan curiosamente los valores de tendencias positivas con significación estadística durante el verano y la primavera.

Las tendencias estacionales de la precipitación en la cuenca del Ebro varían con claridad en el espacio. El invierno es la única estación en la que hemos detectado un descenso generalizado. El descenso es significativo, claro e intenso en el NW (eje Vitoria Pamplona) con tasas que superan $25 \mathrm{~mm} /$ década; algo menos acusado en el Pirineo navarro y Oscense, y muy escaso en el resto de la cuenca. El gradiente global en el espacio queda indicado por una dirección NW-SE a lo largo de la que se dispone la mayor parte de los observatorios con tendencias estadísticamente significativas que solamente representan 1/3 del total de los analizados (Figura 5; Tabla 2).También resulta significativo el descenso en toda la margen oriental de la cuenca aunque de menor intensidad que en la cabecera.

En primavera, verano y otoño, por el contrario, el comportamiento espacial de las tendencias de la precipitación durante la segunda mitad del siglo XX es más complejo (Figura 5 y 6).

Durante la primavera las precipitaciones han descendido significativamente en el Pirineo oscense y leridano y sus somontanos, mientras que en el sector navarro y el conjunto de la cabecera de la cuenca los resultados indican un aumento ligero y generalizado, ocasionalmente intenso en la Sierra de la Demanda y al norte de Vitoria con valores que llegan a $5-10 \mathrm{~mm} /$ década. En amplios sectores de la Ibérica, en el bajo Ebro y en el sector central las pérdidas o ganancias han sido muy escasas y se configura un amplio espacio sin definición clara con tendencias no significativas.

Un patrón semejante se observa en verano, incluso reforzando la magnitud de las pérdidas en el Pirineo centro este con un gradiente N-S hasta el centro de la cuenca. Las ganancias se observan de nuevo en el extremo NW de la cuenca pero en un sec- 


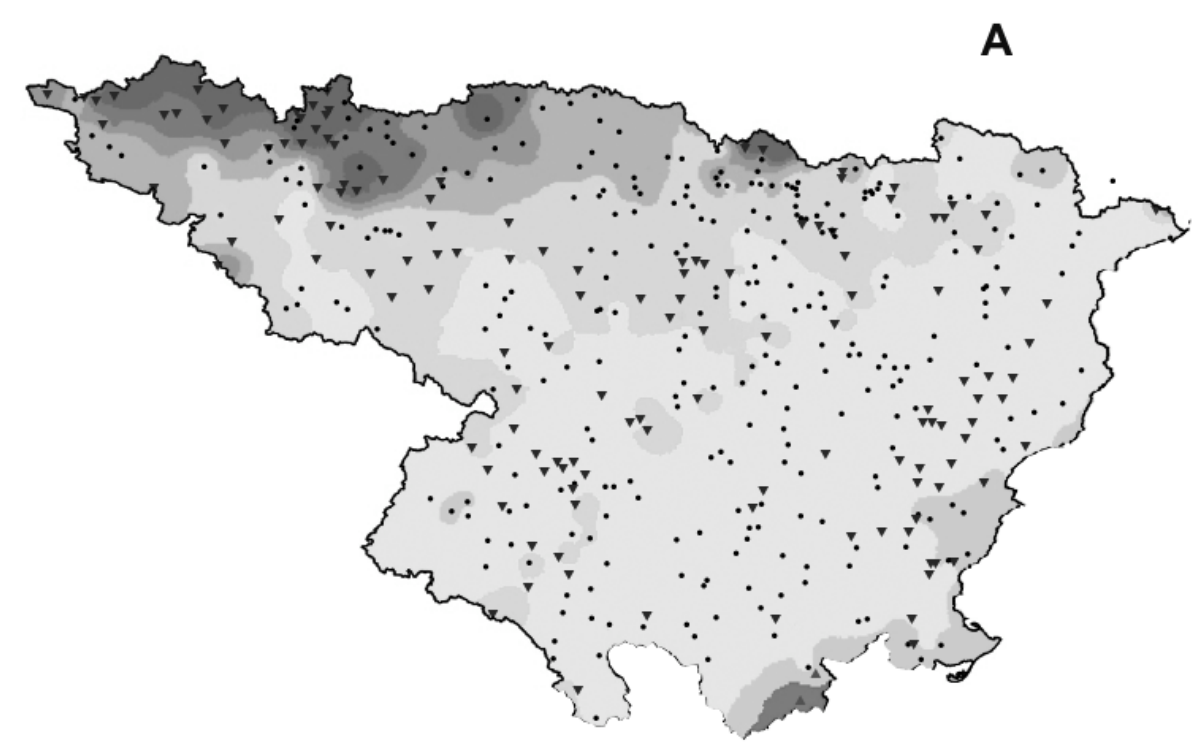

B

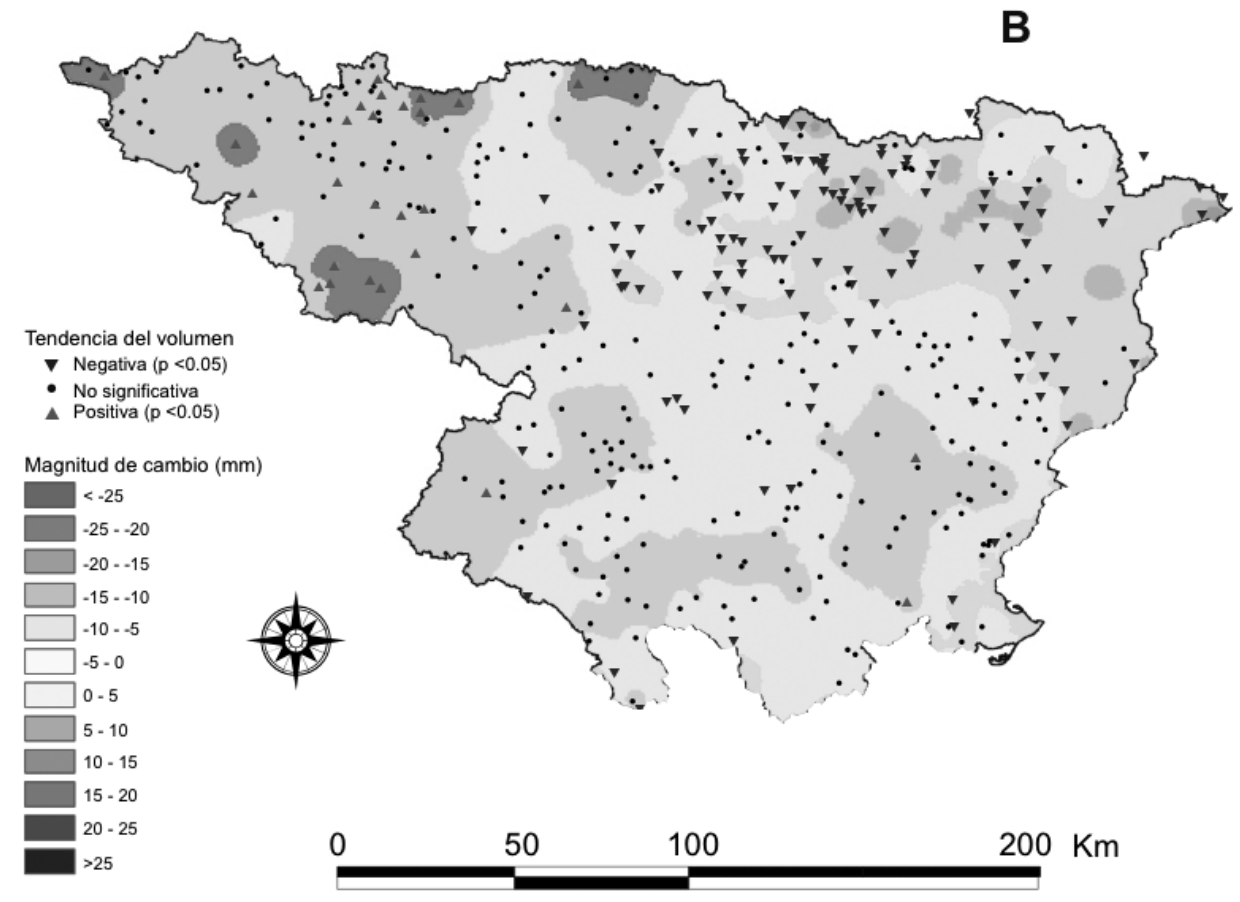

Figura 5. Tendencias de precipitación durante el invierno y la primavera. Los valores interpolados indican la tasa de variación por década a partir del modelo de regresión. 

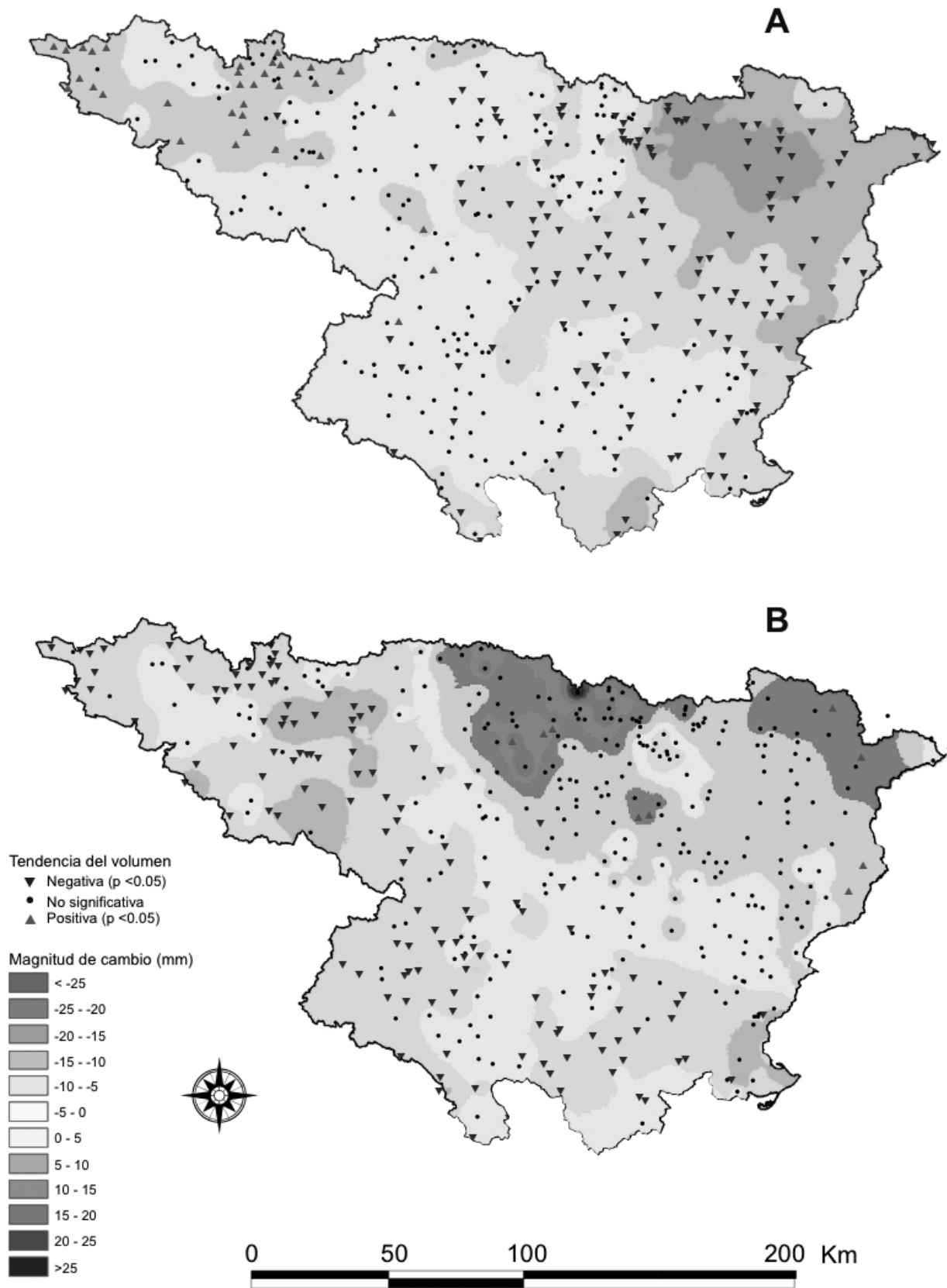

Figura 6. Tendencias de precipitación durante el verano y el otoño. Los valores interpolados indican la tasa de variación por década a partir del modelo de regresión. 
tor muy limitado y con menor intensidad que en primavera. El centro y Sur de la cordillera Ibérica de nuevo no ofrece un comportamiento claro, las tendencias no son significativas aunque las precipitaciones parecen haber descendido.

El otoño muestra un cambio radical respecto a las tendencias estacionales de invierno, primavera y verano. En general, todo el sector montañoso norte (Pirineo Navarro, Oscense y Leridano) ha ganado precipitación, incluso con núcleos de incremento por encima de $20-25 \mathrm{~mm} /$ década. Igualmente las Sierras Exteriores han ganado precipitación, aunque en menor medida, mientras que en todo el eje central, y en la margen derecha las precipitaciones han disminuido. Si bien la tasa de descenso no es muy acusada, sin embargo destaca enormemente el numeroso grupo de observatorios cuyas tendencias son estadísticamente significativas, lo que confirma con mayor vigor el descenso de las precipitaciones en este sector.

Como resumen global, las tendencias anuales de precipitación muestran un predominio de valores negativos. Destacan especialmente dos núcleos de disminución más acusados en el norte, eje Pamplona Vitoria, y el Pirineo oscense oriental y el leridano. Pese a ello no debe olvidarse que en un numeroso grupo de observatorios no se han detectado tendencias significativas e incluso que existen núcleos en los que se han detectado ligeras variaciones al alza de la precipitación anual, lo que reafirma de nuevo la elevada variabilidad del comportamiento de este elemento del clima en la zona de estudio. Los valores más generalizado de disminución en el conjunto de la cuenca suponen pérdidas en torno a 10-30 mm por década (Figura 7).

\section{Discusión}

Durante la segunda mitad el siglo XX se ha detectado una disminución de los totales anuales en el mediterráneo oriental (Giuffrida y Conte, 1989; Maheras y Kolyva Machera, 1990; Amanatidis et al., 1993; Kutiel et al., 1996; Feidas et al., 2007), en el sector central (Piervitali et al., 1998; Delitala et al., 2000; Brunetti et al., 2001 a y b), y en el occidente (Esteban Parra et al., 1998, 2000; De Luis et al., 2000). Este descenso de las precipitaciones anuales se ha relacionado con el incremento global de las temperaturas y los cambios de los gradientes de presiones observados entre Azores e Islandia asociados a las oscilaciones del Atlántico Norte (Houghton et al., 2001). Por su parte, estacionalmente diversos estudios han señalado una disminución de la precipitación de invierno y verano y un incremento de la de primavera, (Bradley et al., 1987, 1989; Maheras, 1988; Díaz et al., 1989). Sin embargo la densidad espacial que todos los trabajos han manejado ha sido baja, de modo que la variabilidad espacial o las transiciones entre sectores no han sido identificadas con precisión. Aún más, la 


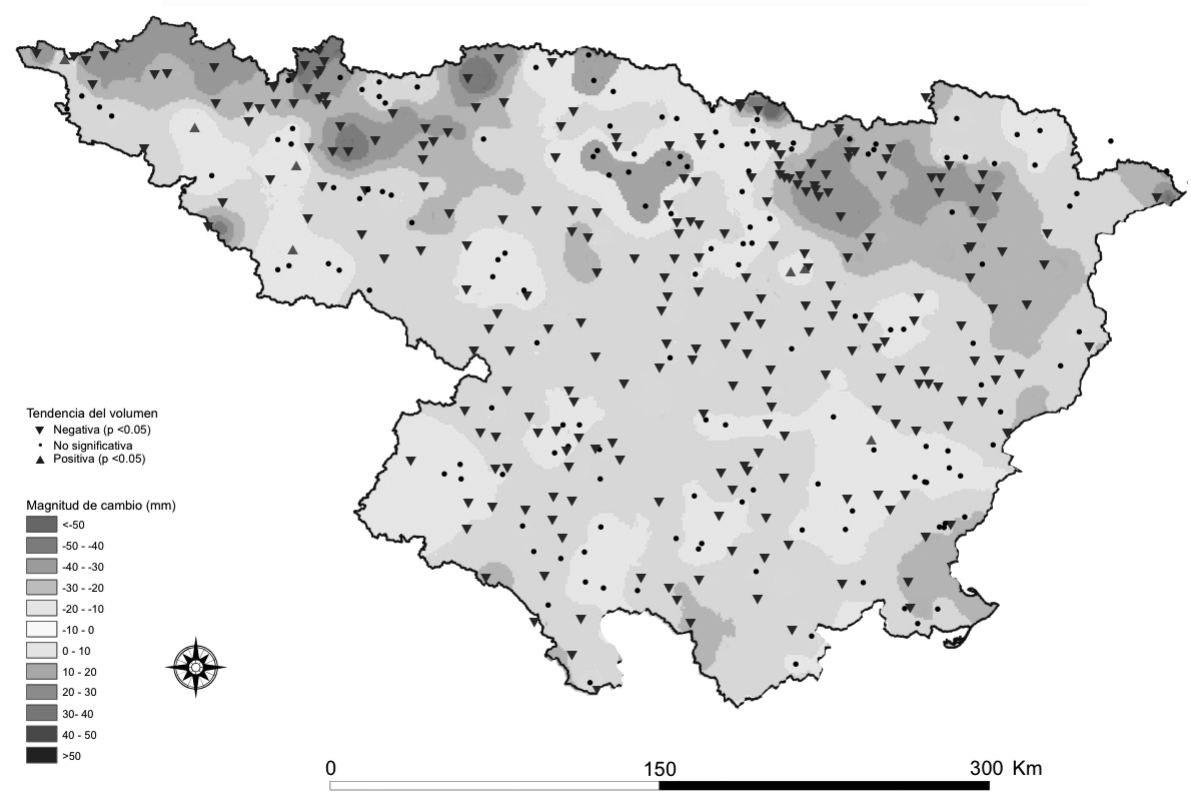

Figura 7. Tendencias de precipitación anual. Los valores interpolados indican la tasa de variación por década a partir del modelo de regresión.

revisión más reciente en la cuenca mediterránea concluye sin detectar una tendencia significativa clara durante el periodo 1951-2000 y nuevamente manifiesta que la variabilidad espacial es la característica esencial de las precipitaciones en el entorno del mar Mediterráneo (Norrant y Douguédroit, 2006).

Las precipitaciones son "el elemento del clima más importante de España, tanto desde el punto de vista climático como en cuanto a recurso, dadas su modesta cuantía en gran parte del territorio y su elevada variabilidad temporal y espacial" (de Castro et al., 2006, p. 9). Este hecho ya había llevado a Sumner et al. (2001) a sugerir años antes la necesidad imperiosa de desarrollar estudios detallados sobre las precipitaciones en la España mediterránea, con lo que ha coincidido plenamente el reciente AR4. Sin embargo, los estudios de detalle, necesarios sobre todo en ambientes con precipitaciones escasas e irregulares, tropiezan con la ausencia generalizada de información climática suficiente que permita lograr conclusiones espacialmente detalladas (De Castro et al., 2006). 
En la Península Ibérica los estudios globales por lo general han empleado menos de 50 observatorios en su mayor parte localizados por debajo de $750 \mathrm{~m}$ de altitud. En su conjunto estas investigaciones no identifican tendencias claras, generalizadas y significativas a lo largo del siglo XX (Esteban Parra et al., 1998; Serrano et al., 1999b; González Rouco et al., 2001; etc), como tampoco en su segunda mitad, particularmente durante el normal 1961-2000 (Mosmannn et al., 2004; Rodrigo y Trigo, 2007). Estacionalmente sin embargo, estos análisis detectaron un descenso de la precipitación en la costa mediterránea y el interior, junto a un suave incremento en el norte durante el periodo 1880-1992 (Estéban Parra et al. 1998). De manera similar Rodrigo y Trigo (2007) recientemente han identificado un descenso de las precipitaciones durante el invierno, primavera y verano en el sur, y en el norte solamente durante el invierno. Por el contrario González Rouco et al. (2001) entre 1899-1989 observaron el aumento de la precipitación de invierno, junto a un descenso en el centro sur durante la primavera, sin tendencias espacialmente claras en verano, y un predominio de tendencias negativas en otoño. El descenso de primavera, aunque no significativo, se ha detectado también en la meseta central entre 1900-1996 por Galán et al., (1999), así como también en el NE por Saladié et al. (2004). Tampoco se han detectado tendencias a lo largo del siglo XX ni en la costa mediterránea (Lana y Burgueño, 2000), ni en la cuenca del Ebro desde 1920 (Abaurrea et al., 2002).

Los estudios regionales y subregionales de la segunda mitad del siglo pasado también ofrecen una gran variabilidad. No se han encontrado tendencias anuales claras y significativas a lo largo de la costa mediterránea (Romero et al., 1998; Guijarro, 2002), NE y Cataluña (Lana y Burgueño, 2000; Saladié et al., 2002, 2004), meseta norte (Labajo et al., 2001; del Río et al., 2005), sudoeste (García Barrón et al., 2002), y Aragón (Cuadrat et al., 2007).

Estacionalmente en la costa mediterránea Romero et al. (1998) encontraron un descenso durante el invierno durante 1964-1990, pero Gonzalez Hidalgo et al (2002 b) identificaron tendencias positivas y significativas en el periodo 1951-2000 muy afectadas por el comportamiento de los años noventa. Durante la primavera, Galán et al., (1999) en el interior, y Saladié et al. (2002 y 2004) en el NE identificaron tendencias negativas, asociadas al descenso generalizado del mes de marzo señalado en toda la vertiente mediterránea por González Hidalgo et al., (2006 a). Descensos durante el otoño también se han detectado en la costa del golfo de Valencia entre 1961 y 2000 (González Hidalgo et al., 2001), mientras los incrementos de la lluvia de verano han sido señalados en el mismo periodo por Mosmannn et al. (2004) en el interior de la Península. En resumen, los resultados de los estudios precedentes permiten componer una imagen de la extraordinaria variabilidad espacial y temporal del comportamiento de las precipitaciones en la Península Ibérica, motivada tanto por la dispar densidad de observatorios empleados como por la diferente periodicidad de cada investigación. En este contexto se inscriben los resultados del análisis de ten- 
dencias de la precipitación de la cuenca del Ebro que hemos presentado, caracterizados por un ligero descenso generalizado de precipitaciones en el total anual, y una compartimentación del espacio estacionalmente, con tendencias que en muchas ocasiones no alcanzan la significación estadística.

El agua es un recurso fundamental para la vida y cualquier modificación de los regímenes pluviales puede tener profundos impactos en las sociedades y los sistemas naturales (Randall et al., 2007). Por ello los impactos del cambio de un régimen pluvial pueden ser especialmente intensos donde las disponibilidades hídricas están controladas por la escasez de los aportes, por su irregularidad, por la existencia de una fuerte demanda, o la conjunción de todas estas causas, circunstancias que parecen converger en la cuenca del Ebro. Desde 1950 la población de toda la vertiente mediterránea española ha aumentado el $86.2 \%$ pero su reparto ha sido disarmónico: en la costa el aumento ha sido de un 107.3\%, mientras que el interior (principalmente la cuenca del Ebro) solamente de 27.6\%. Aún más, la población en el interior se ha concentrado en unas pocas ciudades continuando el éxodo rural en especial en las zonas de montaña. Como resultado hoy día una tercera parte de la población española vive en la costa mediterránea. Junto a los cambios demográficos se han producido también cambios de la demanda de agua y de la presión sobre los recursos hídricos. Durante toda la segunda mitad del siglo XX aquella se ha incrementado en toda la vertiente mediterránea como consecuencia del incremento de la población y las actividades económicas. Pero este incremento ha sido motivado de manera diferente. En la costa, al aumento de población se suma el incremento por actividades turísticas y agricultura intensiva, mientras en el interior el incremento ha sido originado por los regadíos extensivos. En consecuencia, durante la segunda mitad del siglo XX en la fachada mediterránea se han agravado los problemas relacionados con los recursos hídricos tanto por razones climáticas (tendencias globalmente negativas de los aportes, variabilidad espacial de los mismos) como por factores socioeconómicos. Finalmente en España se ha establecido un debate político, económico y social, al haberse planteado el eventual trasvase de agua desde la cuenca del Ebro a las más meridionales de Júcar y Segura.

Puesto que los recursos hídricos se manifiestan con una importancia económica especial en la Península Ibérica, y particularmente en la cuenca del Ebro, si el descenso de la precipitación (como el observado) se mantuviese durante el siglo XXI (como se predice en los modelos; véase de Castro et al., 2006; Christensen et al., 2007, p. 874-877), podría producir consecuencias importantes en la sociedad en un contexto general de incremento de densidad demográfica fuera de los límites de la propia cuenca. Tales consecuencias pudieran proceder especialmente del cambio del régimen de precipitación, como parece apuntar el diferente comportamiento espacial de las tendencias sobre todo en las zonas de montaña, lugar de captación de los recursos hídricos. Del mismo modo desde una perspectiva puramente ecológica al ser 
el agua el factor limitante por excelencia del desarrollo de la vegetación, los descensos de precipitación podrían afectar la supervivencia de determinados ecosistemas mediterráneos, incrementando a su vez la probabilidad de incendios o erosión del suelo entre otras perturbaciones.

Desde un punto de vista estrictamente climático, los análisis estacionales como el presentado en este trabajo pueden ocultar un comportamiento mensual o diario heterogéneo, pero sin embargo la escala anual o estacional proporciona información relevante en otros contextos, tales como la respuesta de los sistemas naturales, el almacenamiento de agua, o el manejo de los recursos hídricos, información crucial para comprender y tratar de predecir y mitigar los impactos sobre los sistemas naturales, las sociedades y las actividades económicas (Morata et al., 2006). El estudio de la cuenca del Ebro, al emplear una densa base de observaciones, describe en una escala no considerada hasta el momento la variabilidad espacial de las tendencias de las precipitaciones en la escala estacional y permite descubrir patrones no contemplados hasta el momento que deberían considerarse en la gestión del territorio.

\section{Conclusiones}

El análisis de tendencias de las precipitaciones en la cuenca del Ebro durante la segunda mitad del siglo XX, realizado con una densa base de datos, sugiere un ligero descenso generalizado de la precipitación anual. Estacionalmente la variabilidad espacial es el rasgo predominante, dentro de una tónica global de tendencia negativa sin significación estadística en muchos casos. Durante el invierno las áreas afectadas por descensos más claros son el NW, el eje central y la margen izquierda del bajo Segre; en primavera las áreas con descenso de precipitación se trasladan hacia el Pirineo oscense y leridano, expandiéndose hacía los somontanos y en general a toda la margen izquierda durante el verano. Por último la margen derecha presenta tendencia mayoritariamente negativa y significativa durante el otoño, a la inversa que la tendencia detectada en la margen izquierda (aunque predominantemente sin significación estadística).

\section{Agradecimientos}

Los autores agradecen el apoyo financiero prestado por el Ministerio de Educación y Ciencia (CGL 2005-04270) y el Gobierno Regional de Aragón, Programa Grupos de 
Investigación Consolidados, Grupo Clima, Agua, Cambio Global y Sistemas Naturales (Resolución 13 de Abril 2005, BOA 20-04-2005), así como al Instituto Nacional de Meteorología, INM, que proporcionó los datos originales. Del mismo modo los autores agradecen los comentarios de dos revisores anónimos y la detección de errores en el manuscrito original.

\section{Referencias}

Abaurrea, J., Asín, J. y Centelles, A. (2002) Caracterización espacio temporal de la evolución de la precipitación anual en la cuenca del Ebro. En Guijarro, J.A. Grimalt, M., Laita, M. y Alonso, S. (eds) El agua y el clima, Publicaciones AEC, Ser A, 3, p. 113-124.

Alexandersson, H. (1986). A homogeneity test applied to precipitation data. Joumal of Climatology, 6, 661-675.

Alexandersson, H. (2001). Homogeneization of climate data, difficult but necessary. En Bruned, M. and López, A.D. (eds) Detecting and modelling Regional climate change and associated impacts, Berlín, Springer Verlag, p. 3-12.

Amanatidis, G.T., Paliatsos, A.G., Repapis, C.C., Bartzis, J.G. (1993). Decreasing precipitation trend in the Marathon area, Greece. International Journal of Climatology, 13, 191-201.

Auer, 1., Bóhm, R., Jurikovic, A., et al. (2005). A new instrumental precipitation dataset for greater Alpine region for the period 1800 2002. International Journal of Climatology, 25, 139-1166.

Begert, M. Schiegel, T. and Kirchhofer, W. (2005). Homogeneous temperature and precipitation series of Switzerland from 1864 to 2000. International Journal of Climatology, 25, 65-80.

Bradley, R.S., Diaz, H.F., Eischeid, J.K., Jones, P.D., Kelly, P.M. and Goodess, C.M.
(1987). Precipitation fluctuations over Northem Hemisphere land areas since the mid 19th century. Science, 237, 171-175.

Bradley, R.S. and Groisman, P.Ya. (1989). Continental scale precipitation variations in the 20th Century. En Sevruk, B. (ed.) Precipitation Measurement, WMO/IAHS/ ETH Workshop on Precipitation Measurement. St. Moritz, p. 499-503.

Brunetti, M., Colacino, M., Mauger, M. and Nanni, T. (2001 a). Trends in the daily intensity of precipitation in ltaly from 1951 1996. International Journal of Climatology, 21, 299-316.

Brunetti, M., Maugeri, M. and Nanni, T. (2001 b). Changes in total precipitation, rainy days and extreme events in northeastern ltaly. International Journal of Climatology, 21, 861-871.

Brunetti, M., Buffoni, L., Maugeri, M. and Nanni, T. (2006). Temperature and precipitation variability in ltaly in the last two centuries from homogeneised instrumental time series. International Journal Climatology, 26, 345-381.

Cuadrat, J.M., Saz, M.A., Vicente, S.M. and González Hidalgo, J.C. (2007). Water resources and precipitation trends in Aragón. Water Resources Development, 23 , 107-123.

Christensen, J.H., Hewitson, B., Busuioc, A. et al. (2007). Regional Climate Projections. En Solomon, S., Qin, D., Manning, M., 
Chen, Z., Marquis, M., Averyt, K.B., Tignor M. and Miller H.L. (eds.) Climate Change 2007. The Physical Science Basis. Contribution of Working Group 1 to the Fourth Assessment Report of the Intergo-vernmental Panel on Climate Change Cambridge University Press, Cambridge and NY, 848-940.

De Castro, M., Martín Vide, J. y Alonso, S. (2006). El clima de España: pasado, presente y escenarios de clima para el siglo XXI. En Impactos del cambio climático en España, Ministerio Medio Ambiente, Madrid.

De Luis, M., Raventós, J., González Hidalgo, J.C., Sánchez, J.R., Cortina, J. (2000). Spatial analysis of rainfall trends: a case study in Valencia Region (E Spain). International Journal Climatology, 20, 1451-1469.

De Luis, M., González Hidalgo, J.C., López Bustins, J.A., Martín Vide, J., Brunetti, M., Nanni, T. and Stepanek, P. (2007). Spatial overlapping areas of four teleconnection indices in Mediterranean façade of Spain. Geophysical Research Abstract, 9, SreND 1606 7962/gra/EGU2007 A 02219.

Del Río, S., Peñas, A. and Fraile, R. (2005). Analysis of recent climatic variations in Castile and Leon (Spain). Atmospheric Research, 73, 69-85.

Delitala, A., Cesari, D., Chesa, P. and Ward, M (2000). Precipitation over Sardinia (ltaly) during the 19461993 rainy seasons and associated large scale climatic variations. International Journal of Climatology, 20, 519-541.

Díaz, H.F., Bradley, R.S. and Eischeid, J.K (1989). Precipitation fluctuations over global land areas since the late 1800s. Journal of Geophysical Research, 94, 1195-1210.

Esteban Parra, M., Rodrigo, F. and Castro, Y. (1998). Spatial and temporal patterns of precipitation in Spain for the period 1880 1992. International Journal Climatology, 18, 1557-1574.
Feidas, H., Noulopoulou, C., Makrogiannis, T. and Bora Senta, E. (2007). Trend analysis of precipitation time series in Greece and their relationship with circulation using surface and satellite data: 19552001. Theoretical and Applied Climatology, 87, 155-177.

Galán, E., Cañada, R., Rasilla, D., Fernández, F. y Cervera, B. (1999). Evolución de las precipitaciones anuales en la Meseta Meridional durante el siglo XX. En Raso, J.M. y Martín Vide, J. (eds) La climatología en España en los albores del siglo XXI, Publicaciones AEC, Ser A 1, p. 169-180.

García Barrón, L., González, M., García, P. y Sousa, A. (2002). Evolución pluviométrica en el suroeste peninsular: variabilidad y disparidad. En García Cordón, J.C., Diego, C, Fdez de Arroyabe P., Garmendia, C y Rasilla, D. (eds) El Clima entre el mar y la montaña, Publicaciones AEC, Serie A, 4, p. 283-290.

Giuffrida, A. and Conte, M. (1989). Long term evolution of the ltalian climate outlined by using the Standardized Anomaly Index (SAI). Proceeding of the Conference on Climate and Water, (1) Helsinki: 197-208.

González Hidalgo, J.C., de Luis, M., Raventós, J. and Sánchez, J.R. (2001). Spatial distribution of seasonal rainfall trends in a westem mediterranean area. International Journal of Climatology, 21, 843-860.

González Hidalgo, J.C., de Luis, M., Stepánek, P., Raventós, J. and Cuadrat, J.M. (2002). Reconstrucción, estabilidad y proceso de homogeneizado de series de precipitación en ambientes de elevada variabilidad pluvial. En Cuadrat, J.M., Vicente, S. y Saz, M.A. (eds) La información climática como berramienta de gestión ambiental, $A G E$ Universidad de Zaragoza, p. 47-57.

González Hidalgo, J.C., de Luis, M. y Raventós J. (2002 b). Variaciones estacionales de la precipitacion en la costa este peninsular durante la decada de los años noventa. En 
Guijarro, J.A. Grimalt, M., Laita, M. y Alonso, S. (eds) El agua y el clima, Publicaciones AEC, Serie A, 3, p. 229-236.

González Hidalgo, J.C., de Luis, M., Stepanek, P. y Lanjeri S. (2006). Propuesta metodológica para realizar un control de calidad de precipitaciones mensuales en la vertiente mediterránea de la Península Ibérica. En Cuadrat, J.M., Saz, M.A., Vicente, S., Lanjeri, S., de Luis, M. y González Hidalgo, J.C. (eds) Clima Sociedad y Medio Ambiente, Publicaciones AEC, Serie A, 5 , p. 591-409.

González Hidalgo, J.C., de Luis, M., Martín Vide, J., López Bustins, J.A., Stepanek, P., Brunetti, M. and Nanni, T. (2007). Spatial overlaping areas of several teleconnection índices on Spain's Mediterranean façade according to autumn rainfall. EMS 7 ECAM8 Abstract, vol 4, EMS2007 A $\neg 00190$.

González Rouco, J.F., Jiménez, J.L., Quesada, V. and Valero, F. (2001). Quality control and homogeneity of precipitation data in Southwest of Europe. International Journal of Climatology, 14, 964-978.

Goodess CM, Jones PD. 2002. Links between circulation and changes in the characteristics of lberian rainfall. International. Journal of Climatology, 22, 1593-1615.

Guijarro, J.A. (2002). Tendencias de la precipitación en el litoral mediterráneo español. En Guijarro, J.A. Grimalt, M., Laita, M. y Alonso, S. (eds) El agua y el clima, Publicaciones de la AEC, Ser A, 3, 237-246.

Houghton, J.T., Ding Y., Griggs D.J., Noguer M., van der Linden P.J. and Xiaosu D. (Eds) (2001). Climate change 2001, The scientific basis. Cambridge University Press, UK

Huntington, T.G. (2006). Evidence for intensification of the global water cycle: Review and synthesis. Journal of Hydrology, 319, 83-95.
Keiser, D. and Griffiths, J. (1997). Problems associated with homogeneity testing in climate variations study: a case study of temperature in the northern Great Plains USA. International Journal Climatology, 17, 497-510.

Khaliq, M.N. and Ouarda, T. (2007). On the critical values of the standard normal homogeneity test (SNHT). International Journal of Climatology, 27, 681-687.

Kutiel, H., Maheras, P. and Guika, S. (1996). Circulation and extreme rainfall conditions in the eastem Mediterranean during the last century. International Journal of Climatology, 16, 73-92.

Labajo, J.L. and Piorno, A. (2001). Regionalization of precipitation in Castilla and Leon (Spain). Analysis of its temporal behaviour. En Bruned, M. and López, A.D. (eds) Detecting and modelling Regional climate change and associated impacts, Berlín, Springer Verlag, p. 163-173.

Lana, X. and Burgueno, A. (2000). Some statistical characteristics of monthly and annual pluviometric irregularity for the Spanish Mediterranean coast. Theoretical and Applied Climatology, 65, 79-97.

Lanzante, L.R. (1996). Resistant, robust and non parametric techniques for the analysis of climate data: theory and examples including applications to historical radiosonde station data. International Journal Climatology, 16, 1197-1226.

Lavorel, S., Canadell, J., Rambal, S. and Terradas J. (1998). Mediterranean terrestrial ecosystems: research priorities on global change effect. Global Ecology and Biogeography Letters, 7, 157-166.

Lionello, P., Boscoso, R. and Malanotte Rizzoli, P. (2006) (eds). Mediterranean Climate Variability. Elsevier, Amsterdam.

Maheras, P. and Kolyva Machera, F. (1990). Temporal and spatial characteristics of annual precipitation over the Balkans in 
the Twentieth Century. International Journal of Climatology, 10, 495-504.

Maheras, P. (1988). Changes in precipitation conditions in the Westem Mediterranean over the last century. Journal of Climate, 8, 179-189.

Martin Vide, J. (2004). Spatial distribution of a daily precipitation concentration index in peninsular Spain. International Journal of Climatology, 24, 959-971.

Millán, T. (1996). Variaciones seculares de las precipitaciones en España. Tesis Doctorado, Universidad de Barcelona. Inédita.

Mitchell, T.D. and Jones, P.D. (2005). An improved method of constructing a database of monthly climate observations and associated high resolution grids. International Journal of Climatology 25, 693-712.

Mosmann, V., Castro, A., Fraile, R., et al. (2004). Detection of statistically significant trends in the summer precipitation of mainland Spain. Atmospheric Research, 70, 43-53.

Munoz Díaz, D. and Rodrigo, F.S. (2004) Spatio temporal patterns of seasonal rainfall in Spain $(1912 \neg 2000)$ using cluster and principal component analysis: comparison. Annales Geophysicae, 22, 1435-1448.

New, M., Todd, M., Hulme, M.and Jones, P. (2001). Precipitation measurements and trends in the twentieth century. International Journal of Climatology, 21, 18991922.

Norrant, C. and Douguedroit, A. (2006). Monthly and daily precipitation trends in the Mediterranean (1950 2000). Theoretical and Applied Climatology, 83, 89-106.

Peterson, T. and Easterling, D. (1994). Creation of homogeneous composite climatological reference series. International Journal of Climatology 14, 671-679

Peterson, T., Easterling, D., Karl, T. et al. (1998 a). Homogeneity adjustments of in situ atmospheric climate data: a review. International Journal of Climatology, 18, 1493-1517.

Peterson, T., Vose, R., Schrnoyer, R. and Razuvaév, V. (1998 b). Global historical climatology network (GHCN) quality control of monthly temperature data. Internatio-nal Journal of Climatology, 18, 1169-1179.

Piervitali, E., Colacino, M., and Conte, M (1998). Rainfall over the Central Westem Mediterranean basin in the period 19511995. Part 1: Precipitation trends. Nuovo Cimento della Societa Italiana di Física $C$ Geophysics \& Space Physics, 21, 331-344.

Randall, D.A., R.A. Wood, S. Bony, et al. (2007). Clímate Models and Their Evaluation. En Solomon, S., Qin, D., Manning, M., Chen, Z., Marquis, M., Averyt, K.B., Tignor M. and Miller H.L. (eds.) Climate Change 2007. The Physical Science Basis. Contribution of Working Group 1 to the Fourth Assessment Report of the Intergovernmental Panel on Climate Change Cambridge University Press, Cambridge and NY, p. 589-662.

Rhoades, D. and Salinger, M. (1993). Adjustment of temperature and rainfall records for site change. International Journal of Climatology, 13, 899-913.

Rodrigo, FS. and Trigo, R.M. (2007). Trends in daily rainfall in the lberian Peninsula from 1951 to 2002. International Journal of Climatology, 27, 513-529.

Rodríquez, R., Llasat, M.C. and Wheeler, D. (1999). Analysis of the Barcelona precipitation series 1850 1991. International Journal of Climatology, 19, 787-801.

Rodríquez Puebla, C., Encinas, A.H., Nieto, S, and Garmendia, J. (1998). Spatial and temporal patterns of annual precipitation variability over the lberian Peninsula. International Journal of Climatology, 18, 299-316. 
Romero, R., Guijarro, J.A. and Alonso, S (1998). A 30 year (1964 1993) daily rainfall data base for the Spanish Mediterranean regions: First exploratory study. International Journal of Climatology, 18, 541-560.

Saladié, O., Brunet, M., Aguilar, E., Sigró, J. y López, D. (2002). Evolución de la precipitación en el sector suroriental de la depresión del Ebro durante la segunda mitad de] siglo XX. En Guijarro, J.A. Grimalt, M., Laita, M. y Alonso, S. (eds) El agua y el clima, Publicaciones de la AEC, Ser A, 3, 335-346.

Saladie, O., Brunet, M., Aguilar, E., Sigró, J. y López, D. (2004). Variaciones y tendencia secular de la precipitación en el sistema Mediterráneo catalán (1901 2000). En García Cordón, J.C., Diego, C, Fdez de Arroyabe P., Garmendia, C y Rasilla, D. (eds) El Clima, entre el mar y la montaña, Publicaciones de la AEC, Ser A 4, p. 399408.

Serrano, A., Mateos, V.L., García, J.A. (1999 b) Trend analysis of monthly precipitation over the lberian Peninsula for the period 1921 1995. Physics and Chemistry of the Earth Part 8 Hydrology Oceans and Atmosphere, 24, 85-90.

Stepánek, P. (2006 a). AnClim software for time series analysis (for Windows 95INT). Dept. of Geography, Fac. of Natural Sciences, MU, Brno, 1.47 MB.
Stepánek, P. (2006 b). ProClimDB software for processing climatological datasets. CHMI, Regional Office Brno.

Sumner, S., Homar, V, and Ramis, C. (2001). Precipitation seasonality in eastern and southern coastal Spain. International Journal of Climatology, 21, 219-247.

Trenberth, K.E., P.D. Jones, P. Ambenje, et al. (2007). Observations: Surface and Atmospheric Climate Change. En Solomon, S., Qin, D., Manning, M., Chen, Z., Marquis, M., Averyt, K.B., Tignor M. and Miller H.L. (eds.) Climate Change 2007. The Physical Science Basis. Contribution of Working Group 1 to the Fourth Assessment Report of the Intergovernmental Panel on Climate Change Cambridge University Press, Cambridge and NY, p. 235-336.

Vincent, L. and Gullet, D. (1999). Canadian historical and homogeneous temperature datasets for climate changes analyses. International Journal of Climatology, 9 , 1375-1388.

Yue, S., Pilon P.P. and Cavadias, G. (2002). Power of the Mann Kendall and Spearman's rho test for detecting monotonic trends in hydrological series. Journal of Hydrology, 259, 254-271. 